\title{
Shi threshold arrangement
}

\author{
Seunghyun Seo \\ Department of of Mathematics Education \\ Kangwon National University \\ Chuncheon 200-701, Republic of Korea \\ shyunseo@kangwon.ac.kr
}

Submitted: Feb 8, 2012; Accepted: Sep 20, 2012; Published: Sep 27, 2012

Mathematics Subject Classifications: 52C35, 05A15

\begin{abstract}
Richard Stanley suggested the problem of finding the characteristic polynomial of a certain hyperplane arrangement defined by $x_{i}+x_{j}=0,1$, which is called the Shi threshold arrangement. We present the answer of the problem, using the finite field method.
\end{abstract}

\section{Introduction}

Much work has been devoted in recent years to studying hyperplane arrangements, especially finding their characteristic polynomials and number of regions. Several authors have worked on computing the number of regions of specific hyperplane arrangements. See for examples [Ath96b, PS00, Ard07].

Stanley's paper [Sta07] on hyperplane arrangements contain classic and more recent results in this field, together with numerous problems. We consider the following problems [Sta07, p. 473] of finding the characteristic polynomial of the following hyperplane arrangement:

$$
x_{i}+x_{j}=0,1, \quad 1 \leqslant i<j \leqslant n .
$$

Stanley called the arrangement by "Shi threshold arrangements"

The present paper solves this problem by the finite field method (see [Ath96b, CR70, Sta07]). In Section 2, we introduce the basic notations of hyperplane arrangements. In Section 3, we find the characteristic polynomial of the Shi threshold arrangement. We also calculate the exponential generating function for the characteristic polynomial of the Shi threshold arrangement. In Section 4, we comment on some related works and further studies. 


\section{Preliminaries}

We recall some of the basic concepts of hyperplane arrangements. For a more thorough introduction, see [OT92, Sta99].

\subsection{Intersection posets and characteristic polynomials}

Given a field $K$ and a positive integer $n$, a hyperplane arrangement of dimension $n$ over $K$ is a finite set of affine hyperplanes in $V=K^{n}$. We will refer to hyperplane arrangements simply as arrangements.

Now, let $V=\mathbb{R}^{n}$. A region of an arrangement $\mathcal{A}$ is a connected component of the complement $X$ of the hyperplanes:

$$
X=\mathbb{R}^{n}-\bigcup_{H \in \mathcal{A}} H
$$

Let $R(\mathcal{A})$ denote the set of regions of $\mathcal{A}$, and let $r(\mathcal{A})$ be the number of regions.

Given an arrangement $\mathcal{A}$ in $V$, let $L(\mathcal{A})$ be the set of all nonempty intersections of hyperplanes in $\mathcal{A}$, including $V$. Define $x \leqslant y$ in $L(\mathcal{A})$ if $x \supseteq y$ as a subsets of $V$. We call $L(\mathcal{A})$ the intersection poset of $\mathcal{A}$. It is easy to show that $L(\mathcal{A})$ is a graded poset with the rank function $\mathrm{rk}: L(\mathcal{A}) \rightarrow \mathbb{N}$ defined by

$$
\operatorname{rk}(x)=\operatorname{dim}(V)-\operatorname{dim}(x)=n-\operatorname{dim}(x),
$$

where $\operatorname{dim}(x)$ is the dimension of $x$ as an affine subspace of $V$.

Given a finite poset $P$ with $\hat{0}$, the Möbious function $\mu: P \rightarrow \mathbb{Z}$ is defined by

$$
\mu(\hat{0})=1 \quad \text { and } \quad \mu(x)=-\sum_{y<x} \mu(y) .
$$

Originally, the Möbious function $\mu$ goes from the set of all intervals of $P$ to $\mathbb{Z}$. But we will only consider the intervals of the form $[\hat{0}, x]$, which are identified with $x \in P$.

Definition 1. The characteristic polynomial $\chi_{\mathcal{A}}(t)$ of the arrangement $\mathcal{A}$ is defined by

$$
\chi_{\mathcal{A}}(t):=\sum_{x \in L(\mathcal{A})} \mu(x) t^{\operatorname{dim}(x)}
$$

The characteristic polynomial plays an important role in the theory of arrangements. One of the important result is as follows.

Theorem 2 (Zaslavsky [Zas75]). For any arrangement $\mathcal{A}$ in $\mathbb{R}^{n}$, we have

$$
r(\mathcal{A})=(-1)^{n} \chi_{\mathcal{A}}(-1)
$$




\section{$2.2 \quad$ Finite field method}

In general, given an arrangement $\mathcal{A}$, it is hard to compute the characteristic polynomial of $\mathcal{A}$. However, if $\mathcal{A}$ in $\mathbb{Q}^{n}$, (i.e., all coefficients of hyperplanes in $\mathcal{A}$ are rational), then there is a powerful method for computing its characteristic polynomial. Given a prime number $p$, let $\mathbb{F}_{p}$ be the finite field of order $p$. If $H$ is a hyperplane of $\mathcal{A}$ in $\mathbb{Q}^{n}$, by multiplying a proper integer to the equation of $H$, we can regard all the coefficients of the equation of $H$ as integers. In this case we can take coefficients modulo $p$ and get an arrangement $\mathcal{A}_{p}$ in $\mathbb{F}_{p}^{n}$. It is well known that there are all but finitely many primes $p$ such that $L(\mathcal{A})$ is isomorphic to $L\left(\mathcal{A}_{p}\right)$.

Theorem 3 (Crapo, Rota [CR70]; Orlik, Terao [OT92]; Athanasisadis [Ath96b]). Let $\mathcal{A}$ be an arrangement in $\mathbb{Q}^{n}$. If $L(\mathcal{A}) \cong L\left(\mathcal{A}_{q}\right)$ for some prime $q$, then

$$
\chi_{\mathcal{A}}(q)=\left|\mathbb{F}_{q}^{n}-\bigcup_{H \in \mathcal{A}_{q}} H\right|=q^{n}-\left|\bigcup_{H \in \mathcal{A}_{q}} H\right|,
$$

which is called the finite field method.

\subsection{Shi arrangement and threshold arrangement}

Now we consider two special hyperplane arrangements. The Shi arrangement $\mathcal{S}_{n}$ is given by

$$
x_{i}-x_{j}=0,1, \quad 1 \leqslant i<j \leqslant n,
$$

which was first appeared in [Shi86, §7]. Using Poincaré polynomials, Headley [Hea97] found that the characteristic polynomial of $\mathcal{S}_{n}$ is

$$
\chi_{\mathcal{S}_{n}}(t)=t(t-n)^{n-1} .
$$

Athanasiadis [Ath96b] proved it by the finite field method.

Applying Zaslavsky's theorem to the equation (2), we have

$$
r\left(\mathcal{S}_{n}\right)=(n+1)^{n-1} .
$$

Pak and Stanley [Sta98, §4] and Athanasiadis and Linusson [AL99] proved (3) bijectively. They gave bijections from $\mathcal{R}\left(\mathcal{S}_{n}\right)$ to the set of all parking functions on $[n]$.

The threshold arrangement $\mathcal{T}_{n}$ is given by

$$
x_{i}+x_{j}=0, \quad 1 \leqslant i<j \leqslant n .
$$

The notation "threshold" comes from threshold graphs that were introduced by Chvátal and Hammer [CH77]. There is a canonical bijection from $\mathcal{T}_{n}$ to the set of threshold graphs on $[n]$. Therefore the exponential generating function for the number of regions of $\mathcal{T}_{n}$ is given by

$$
\sum_{n \geqslant 0} r\left(\mathcal{T}_{n}\right) \frac{x^{n}}{n !}=\frac{e^{x}(1-x)}{2-e^{x}}
$$


Later, Stanley [Sta07, p. 473] showed that the exponential generating function for the characteristic polynomial of $\mathcal{T}_{n}$ is given by

$$
\sum_{n \geqslant 0} \chi_{\mathcal{T}_{n}}(t) \frac{x^{n}}{n !}=(1+x)\left(2 e^{x}-1\right)^{(t-1) / 2} .
$$

Note that it is open to find a combinatorial interpretation of each coefficient of $\chi_{\mathcal{T}_{n}}(t)$ as the number of certain threshold graphs.

\section{Shi threshold arrangement}

As a generalized threshold arrangement, we consider an arrangement defined by

$$
x_{i}+x_{j}=0,1, \quad 1 \leqslant i<j \leqslant n .
$$

Stanley [Sta07, p. 473] introduced the arrangement and called the Shi threshold arrangement. Let $\mathcal{S T}_{n}$ denote the Shi threshold arrangement. We will find the characteristic polynomial of Shi threshold arrangement and its exponential generating function.

\subsection{Characteristic polynomial of $\mathcal{S T}_{n}$}

From the finite field method, there exist infinitely many odd primes $q=2 r+1$ such that

$$
\chi_{\mathcal{S T}_{n}}(q)=\mid\left\{\left(a_{1}, \ldots, a_{n}\right) \in \mathbb{F}_{q}^{n} \mid a_{i}+a_{j} \neq 0, a_{i}+a_{j} \neq 1 \text {, for } i<j\right\} \mid .
$$

Let $X$ be the set $\left\{\left(a_{1}, \ldots, a_{n}\right) \in \mathbb{F}_{q}^{n} \mid a_{i}+a_{j} \neq 0, a_{i}+a_{j} \neq 1\right.$, for $\left.i<j\right\}$. Clearly we have $|X|=\chi_{\mathcal{S T}_{n}}(q)$. Given a sequence $\left(u_{1}, \ldots, u_{q}\right)=(0,1,-1,2,-2, \ldots, r,-r)$ of $\mathbb{F}_{q}$, let $Y$ be the set of all $n$-tuples $\left(b_{1}, \ldots, b_{n}\right) \in \mathbb{F}_{q}^{n}$ satisfying the following conditions:

- $\left|\left\{i \in[n] \mid b_{i}=0\right\}\right| \leqslant 1$ and $\left|\left\{i \in[n] \mid b_{i}=-r\right\}\right| \leqslant 1$.

- If $u_{j} \in\left\{b_{i} \mid i \in[n]\right\}$ for some $j \in[q]$, then $\left\{u_{j-1}, u_{j+1}\right\} \cap\left\{b_{i} \mid i \in[n]\right\}=\emptyset$.

It is obvious that $X$ and $Y$ are the same set which depends on $q$. Thus, to give $\chi_{\mathcal{S} \mathcal{T}_{n}}(q)$, it suffices to find the cardinality of $Y$.

Lemma 4. For positive integers $m$ and $n$, let $\mathbf{u}=\left(u_{1}, \ldots, u_{m}\right)$ be a sequence of distinct $m$ elements. From the set $\left\{u_{1}, \ldots, u_{m}\right\}$, choose $n$-tuples $\left(b_{1}, \ldots, b_{n}\right)$ in $\left\{u_{1}, \ldots, u_{m}\right\}^{n}$ such that no consecutive elements can be chosen. Then the number of such ways is

$$
\sum_{1 \leqslant j \leqslant \min \left(n,\left\lfloor\frac{m+1}{2}\right\rfloor\right)}\left(\begin{array}{c}
m-j+1 \\
j
\end{array}\right) S(n, j) j !,
$$

where $S(n, j)$ is the Stirling number of the second kind. 
Proof. let $Z$ be the set of all functions $f:[n] \rightarrow[m]$ such that the inverse images $f^{-1}(\{i\})$ or $f^{-1}(\{i+1\})$ are empty for all $i=1, \ldots, m-1$. Correspond an element $\left(b_{1}, \ldots, b_{n}\right)$ satisfying the condition to a function $f \in Z$ via $f(i):=j$, where $b_{i}=u_{j}$. It is obvious that the correspondence indeed a bijection. If $f \in Z$ then the image $f([n])=A$ is the subset of $[m]$ such that no consecutive numbers belong to $A$. Thus the number of selecting such $A$ is equal to $\left(\begin{array}{c}m-j+1 \\ j\end{array}\right)$. Once $A$ is chosen, the number of surjective functions from $[n]$ to $A$ is equal to $j ! S(n, j)$, which completes the equation (7).

For convention we allow $j=0$ in $(7)$, because $S(n, 0)=0$ if $n>0$. For nonnegative integers $m$ and $n$, let $a_{m}(n)$ be

$$
a_{m}(n):=\sum_{0 \leqslant j \leqslant n}\left(\begin{array}{c}
m-j+1 \\
j
\end{array}\right) S(n, j) j !,
$$

where $S(n, k)$ is the Stirling number of the second kind. Now we go back to Shi threshold arrangements, i.e.,

$$
\mathbf{u}=\left(u_{1}, \ldots, u_{q}\right)=(0,1,-1,2,-2, \ldots, r,-r) .
$$

To enumerate the set $Y$, we should regard the additional condition: Each 0 and $-r$ can be chosen at most once. So we have three cases to consider.

1. Neither 0 nor $-r$ are selected.

2. Either 0 or $-r$ is selected.

3. Both 0 and $-r$ are selected.

By Lemma 4, the number of elements $\left(b_{1}, \ldots, b_{n}\right)$ of the first case is

$$
\sum_{0 \leqslant j \leqslant \min (n, r)}\left(\begin{array}{c}
2 r-j \\
j
\end{array}\right) S(n, j) j !,
$$

which becomes $a_{2 r-1}(n)$ for $r \geqslant n$, i.e., for infinitely many sufficiently large primes $q=$ $2 r+1$. Similarly, the second case is $2 n a_{2 r-2}(n-1)$ and the third case is $n(n-1) a_{2 r-3}(n-2)$ for infinitely many sufficiently large primes $q=2 r+1$. Thus we have the following result:

Theorem 5. The characteristic polynomial of the Shi-threshold arrangement $\mathcal{S T}_{n}$ is given by

$$
\begin{aligned}
\chi_{\mathcal{S} \mathcal{T}_{n}}(t)= & \sum_{j \geqslant 0}(t-j-1)_{j} S(n, j)+2 n \sum_{j \geqslant 0}(t-j-2)_{j} S(n-1, j) \\
& +n(n-1) \sum_{j \geqslant 0}(t-j-3)_{j} S(n-2, j)
\end{aligned}
$$

where $(x)_{k}$ is defined by $(x)_{0}=1$ and $(x)_{k}=x(x-1) \cdots(x-k+1)$ for $k \geqslant 1$. 
For instance $\chi_{\mathcal{S} \mathcal{T}_{0}}(t)=1, \quad \chi_{\mathcal{S} \mathcal{T}_{1}}(t)=t, \quad \chi_{\mathcal{S} \mathcal{T}_{2}}(t)=t^{2}-2 t$, and

$$
\begin{aligned}
& \chi_{\mathcal{S T}_{3}}(t)=t^{3}-6 t^{2}+12 t-8 \\
& \chi_{\mathcal{S T}_{4}}(t)=t^{4}-12 t^{3}+60 t^{2}-142 t+130 \\
& \chi_{\mathcal{S T}_{5}}(t)=t^{5}-20 t^{4}+180 t^{3}-870 t^{2}+2190 t-2252
\end{aligned}
$$

Applying the Zaslovsky's Theorem, we have the followings.

Corollary 6. The number of regions in the Shi-threshold arrangement $\mathcal{S T}_{n}$ is given by

$$
\begin{aligned}
r\left(\mathcal{S T}_{n}\right)= & \sum_{j \geqslant 0}\left(\begin{array}{c}
2 j+1 \\
j
\end{array}\right) j !(-1)^{n-j} S(n, j)+2 n \sum_{j \geqslant 0}\left(\begin{array}{c}
2 j+2 \\
j
\end{array}\right) j !(-1)^{n-j} S(n-1, j) \\
& +n(n-1) \sum_{j \geqslant 0}\left(\begin{array}{c}
2 j+3 \\
j
\end{array}\right) j !(-1)^{n-j} S(n-2, j) .
\end{aligned}
$$

The sequence $\left\{r\left(\mathcal{S} \mathcal{T}_{n}\right)\right\}_{n \geqslant 0}$ starts with

$1,1,3,27,345,5513,106619,2426819, \ldots$

which is not listed in the "Online Encyclopedia of Integer Sequences".

\subsection{Generating function for $\chi_{\mathcal{S} \mathcal{T}_{n}}(t)$}

Recall the definition of $a_{m}(n)$ in equation (8). By exponential formula we have

$$
a_{m}(n)=\sum_{j \geqslant 0}\left(\begin{array}{c}
m-j+1 \\
j
\end{array}\right)\left[\frac{x^{n}}{n !}\right]\left(e^{x}-1\right)^{j},
$$

where $\left[\frac{x^{n}}{n !}\right] F(x)$ is the coefficient of $\frac{x^{n}}{n !}$ in the power series $F(x)$. Thus

$$
\sum_{n \geqslant 0} a_{m}(n) \frac{x^{n}}{n !}=\sum_{j \geqslant 0}\left(\begin{array}{c}
m-j+1 \\
j
\end{array}\right)\left(e^{x}-1\right)^{j} .
$$

It is well known (for example [Wil94, p. 54]) that

$$
\sum_{j \geqslant 0}\left(\begin{array}{c}
2 j+\alpha \\
j
\end{array}\right) z^{j}=\frac{C(z)^{\alpha}}{\sqrt{1-4 z}}
$$

where $C(z)$ is the generating function for the Catalan number, i.e.,

$$
C(z)=\frac{1-\sqrt{1-4 z}}{2 z}=\sum_{n \geqslant 0} \frac{1}{n+1}\left(\begin{array}{c}
2 n \\
n
\end{array}\right) z^{n} .
$$


Substitute $\alpha=-m-2$ and $z=-\left(e^{x}-1\right)$ then

$$
\sum_{j \geqslant 0}\left(\begin{array}{c}
2 j+\alpha \\
j
\end{array}\right) z^{j}=\sum_{j \geqslant 0}\left(\begin{array}{c}
m-j+1 \\
j
\end{array}\right)\left(e^{x}-1\right)^{j} .
$$

Combining (9), (10), and (11) yields that

$$
\sum_{n \geqslant 0} a_{m}(n) \frac{x^{n}}{n !}=\frac{C\left(1-e^{x}\right)^{-m-2}}{\sqrt{4 e^{x}-3}} .
$$

In Theorem $5, \chi_{\mathcal{S} \mathcal{T}_{n}}(t)$ is expressed by a linear combination of $a_{m}(n)$ 's. So, with simple calculations, we can deduce the exponential generating function for $\chi_{\mathcal{S} \mathcal{T}_{n}}(t)$, which is

$$
\sum_{n \geqslant 0} \chi_{\mathcal{S T}_{n}}(t) \frac{x^{n}}{n !}=\frac{C\left(1-e^{x}\right)^{-t}}{\sqrt{4 e^{x}-3}}\left(x C\left(1-e^{x}\right)+1\right)^{2} .
$$

Put $t=-1$ and $x=-x$ in (12) to get

$$
\sum_{n \geqslant 0} r\left(\mathcal{S} \mathcal{T}_{n}\right) \frac{x^{n}}{n !}=\frac{C\left(1-e^{-x}\right)}{\sqrt{4 e^{-x}-3}}\left(x C\left(1-e^{-x}\right)-1\right)^{2}
$$

Unfortunately, it looks like that right hand sides of equations (12) and (13) cannot be simplified.

\section{Remarks}

A similar problem was solved by Athanasiadis in his $\mathrm{PhD}$ thesis [Ath96a, Cor 7.3.3], about the arrangement defined by

$$
x_{i}+x_{j}=0,1 \quad 1 \leqslant i \leqslant j \leqslant n .
$$

The difference between this arrangement and the Shi-threshold arrangement is whether $i \leqslant j$ or $i<j$. In fact, the arrangement (14) is exponentially stable [Ath96a, p. 97], but the Shi-threshold arrangement is not, so we cannot apply his method in [Ath96a, Thm 7.3.2].

Ardila [Ard07, Thm 4.5] computed the Tutte polynomials of various arrangements including the threshold arrangement. It would be interesting to find the Tutte polynomial of Shi threshold arrangement.

Finally consider a "generalized" threshold arrangement such as

$$
x_{i}+x_{j}=-l,-l+1, \ldots, m-1, m \quad 1 \leqslant i \leqslant j \leqslant n .
$$

It would be desirable to find the characteristic polynomial or the Tutte polynomial for the arrangement (15). 


\section{Acknowledgements}

We thank the anonymous referee for valuable comments and suggestions to improve this article. This research was supported by the Basic Science Research Program through the National Research Foundation of Korea funded by the Ministry of Educations, Science and Technology (2012-0004476).

\section{References}

[AL99] Christos A. Athanasiadis and Svante Linusson. A simple bijection for the regions of the Shi arrangement of hyperplanes. Discrete Math., 204(1-3):27-39, 1999.

[Ard07] Federico Ardila. Computing the Tutte polynomial of a hyperplane arrangement. Pacific J. Math., 230(1):1-26, 2007.

[Ath96a] Christos A. Athanasiadis. Algebraic combinatorics of graph spectra, subspace arrangements and Tutte polynomials. 1996. Thesis (Ph.D.)-Massachusetts Institute of Technology.

[Ath96b] Christos A. Athanasiadis. Characteristic polynomials of subspace arrangements and finite fields. Adv. Math., 122(2):193-233, 1996.

[CH77] Václav Chvátal and Peter L. Hammer. Aggregation of inequalities in integer programming. In Studies in integer programming (Proc. Workshop, Bonn, 1975), pages 145-162. Ann. of Discrete Math., Vol. 1. North-Holland, Amsterdam, 1977.

[CR70] Henry H. Crapo and Gian-Carlo Rota. On the foundations of combinatorial theory: Combinatorial geometries. The M.I.T. Press, Cambridge, Mass.-London, preliminary edition, 1970.

[Hea97] Patrick Headley. On a family of hyperplane arrangements related to the affine Weyl groups. J. Algebraic Combin., 6(4):331-338, 1997.

[OT92] Peter Orlik and Hiroaki Terao. Arrangements of hyperplanes. Springer-Verlag, Berlin, 1992.

[PS00] Alexander Postnikov and Richard P. Stanley. Deformations of Coxeter hyperplane arrangements. J. Combin. Theory Ser. A, 91(1-2):544-597, 2000.

[Shi86] Jian Yi Shi. The Kazhdan-Lusztig cells in certain affine Weyl groups, volume 1179 of Lecture Notes in Mathematics. Springer-Verlag, Berlin, 1986.

[Sta98] Richard P. Stanley. Hyperplane arrangements, parking functions and tree inversions. In Mathematical essays in honor of Gian-Carlo Rota (Cambridge, MA, 1996), volume 161 of Progr. Math., pages 359-375. Birkhäuser Boston, Boston, MA, 1998.

[Sta99] Richard P. Stanley. Enumerative combinatorics. Vol. 2. Cambridge University Press, Cambridge, 1999. 
[Sta07] Richard P. Stanley. An introduction to hyperplane arrangements. In Geometric combinatorics, volume 13 of IAS/Park City Math. Ser., pages 389-496. Amer. Math. Soc., Providence, RI, 2007.

[Wil94] Herbert S. Wilf. generatingfunctionology. Academic Press Inc., Boston, MA, second edition, 1994.

[Zas75] Thomas Zaslavsky. Facing up to arrangements: face-count formulas for partitions of space by hyperplanes. Mem. Amer. Math. Soc., 1(issue 1, 154):vii+102, 1975 . 